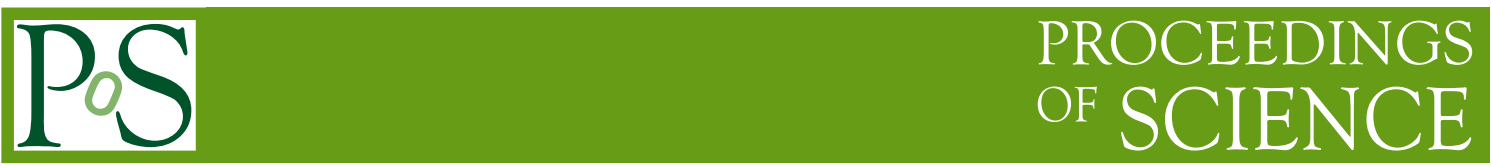

\title{
Unitarity-Cuts, Stokes' Theorem and Berry's Phase
}

\author{
Pierpaolo Mastrolia*† \\ Centro Studi e Ricerche "E. Fermi”, Piazza del Viminale 1, I-00184, Roma, Italy \\ Dipartimento di Fisica, Università di Salerno, Via Ponte don Melillo, I 84084 Fisciano, Italy \\ Theory Department, CERN, CH-1211 Geneva 23, Switzerland \\ E-mail: pierpaolo.mastrolia@cern.ch
}

Two-particle unitarity-cuts of scattering amplitudes can be efficiently computed by applying Stokes' Theorem, in the fashion of the Generalised Cauchy Theorem. Consequently, the Optical Theorem can be related to the Berry Phase, showing how the imaginary part of arbitrary one-loop Feynman amplitudes can be interpreted as the flux of a complex 2-form.

RADCOR 2009 - 9th International Symposium on Radiative Corrections (Applications of Quantum Field Theory to Phenomenology),

October 25 - 302009

Ascona, Switzerland

\footnotetext{
*Speaker.

$\dagger$ In memory of my friend Thomas (Binoth), who reacted with his characteristic enthusiasm when I first shew him the results now collected in this manuscripts. I acknowledge Tania Robens for clarifying discussions. My participation to the conference was supported by HepTools.
} 


\section{Introduction}

Unitarity and geometric phases are two ubiquitous properties of physical systems. The Berry phase is the phase acquired by a system when it is subjected to a cyclic evolution, resulting only from the geometrical properties of the path traversed in the parameter space because of anholonomy $[1,2]$. Unitarity represents the probability conservation in particle scattering processes described by the unitary scattering operator, $S$. The relation, $S=1+i T$, between the $S$-operator and the transition operator, $T$, leads to the Optical Theorem, $-i\left(T-T^{\dagger}\right)=T^{\dagger} T$. The matrix elements of this equation between initial and final states are expressed, in perturbation theory, in terms of Feynman diagrams. The evaluation of the right hand side requires the insertion of a complete set of intermediate states. Therefore, since $-i\left(T-T^{\dagger}\right)=2 \operatorname{Im} T$, the Optical Theorem yields the computation of the imaginary part of Feynman integrals from a sum of contributions from all possible intermediate states.

The Cutkosky-Veltman rules, implementing the unitarity conditions, allow the calculation of the discontinuity across a branch cut of an arbitrary Feynman amplitude, which corresponds to its imaginary part [3]. Accordingly, the imaginary part of a given Feynman integral can be computed by evaluating the phase-space integral obtained by cutting two internal particles, which amounts to applying the on-shell conditions and replacing their propagators by the corresponding $\delta$-function, $\left(p^{2}-m^{2}+i 0\right)^{-1} \rightarrow(2 \pi i) \delta^{(+)}\left(p^{2}-m^{2}\right)$. In later studies the problem of finding the discontinuity of a Feynman integral associated to a singularity was addressed in the language of homology theory and differential forms [4].

More recently multi-particle cuts have been combined with the use of complex momenta for on-shell internal particles into very efficient techniques, by-now known as unitarity-based methods, to compute scattering amplitudes for arbitrary processes. These methods exploit two general properties of scattering amplitudes like analyticity, granting that amplitudes are determined by their own singularity-structure, and unitarity, granting that the residues at the singular points factorize into products of simpler amplitudes. Unitarity and analyticity become tools for the quantitative determinaton of one-loop amplitudes [5] when merged with the existence of an underlying representation of amplitudes as a combination of basic scalar one-loop functions [6]. These functions, known as Master Integrals (MI's), are $n$-point one-loop integrals, $I_{n}(1 \leq n \leq 4)$, with trivial numerator, equal to 1 , characterised by external momenta and internal masses present in the denominator. In general, the fulfillment of multiple-cut conditions requires loop momenta with complex components. Since the loop momentum has four components, the effect of the cut-conditions is to fix some of them according to the number of the cuts. Any quadruple-cut [11] fixes the loop-momentum completly, yielding the algebraic determination of the coefficients of $I_{n},(n \geq 4)$; the coefficient of 3-point functions, $I_{3}$, are extracted from triple-cut $[12,13,14,15,16,17]$; the evaluation of double-cut $[18,19,20,14,21,16,22,23,17]$ is necessary for extracting the coefficient of 2-point functions, $I_{2}$; and finally, in processes involving massive particles, the coefficients of 1-point functions, $I_{1}$, are detected by single-cut $[16,24,25]$. In cases where fewer than four denominators are cut, the loop momentum is not frozen: the free-components are left over as phase-space integration variables.

In [26, 27], I introduced a novel efficient method for the analytic evaluation of the coefficients of one-loop 2-point functions via double-cuts. Spun-off from the spinor-integration technique [18, $19,20]$, that method is an application of Stokes' Theorem. Due to a special decomposition of the 
loop-momentum, the double-cut phase-space integral is written as parametric integration of rational function in two complex-conjugated variables. By applying Stokes' Theorem, the integration is carried on in two simple steps: an indefinite integration in one variable, followed by Cauchy's Residue Theorem in the conjugated one.

The coefficients of the 2-point scalar functions, being proportional to the rational term of the double-cut, can be directly extracted from the indefinite integration by Hermite Polynomial Reduction.

\section{Double-Cut}

The two-particle Lorentz invariant phase-space (LIPS) in the $K^{2}$-channel is defined as,

$$
\int d^{4} \Phi=\int d^{4} \ell_{1} \delta^{(+)}\left(\ell_{1}^{2}-m_{1}^{2}\right) \delta^{(+)}\left(\left(\ell_{1}-K\right)^{2}-m_{2}^{2}\right),
$$

where $K^{\mu}$ is the total momentum across the cut. We introduce a suitable parametrization for $\ell_{1}^{\mu}$ $[26,20]$, in terms of four massless momenta, which is a solution of the two on-shell conditions, $\ell_{1}^{2}=m_{1}^{2}$ and $\left(\ell_{1}-K\right)^{2}=m_{2}^{2}$,

$$
\ell_{1}^{\mu}=\frac{1-2 \rho}{1+z \bar{z}}\left(p^{\mu}+z \bar{z} q^{\mu}+z \varepsilon_{+}^{\mu}+\bar{z} \varepsilon_{-}^{\mu}\right)+\rho K^{\mu},
$$

where $p_{\mu}$ and $q_{\mu}$ are two massless momenta with the requirements,

$$
p_{\mu}+q_{\mu}=K_{\mu}, \quad p^{2}=q^{2}=0, \quad 2 p \cdot q=2 p \cdot K=2 q \cdot K \equiv K^{2} ;
$$

the vectors $\varepsilon_{+}^{\mu}$ and $\varepsilon_{-}^{\mu}$ are orthogonal to both $p^{\mu}$ and $q^{\mu}$, with the following properties ${ }^{1}$,

$$
\varepsilon_{+}^{2}=\varepsilon_{-}^{2}=0=\varepsilon_{ \pm} \cdot p=\varepsilon_{ \pm} \cdot q, \quad 2 \varepsilon_{+} \cdot \varepsilon_{-}=-K^{2} .
$$

The pseudo-threshold $\rho=\left(K^{2}+m_{1}^{2}-m_{2}^{2}-\sqrt{\lambda}\right) /\left(2 K^{2}\right)$, with $\lambda=\left(K^{2}\right)^{2}+\left(m_{1}^{2}\right)^{2}+\left(m_{2}^{2}\right)^{2}-$ $2 K^{2} m_{1}^{2}-2 K^{2} m_{2}^{2}-2 m_{1}^{2} m_{2}^{2}$, depends only on the kinematics. The complex conjugated variables $z$ and $\bar{z}$ parametrize the degrees of freedom left over by the cut-conditions. Because of (2.2), the LIPS in (2.1) reduces to the remarkable expression,

$$
\int d^{4} \Phi=(1-2 \rho) \iint \frac{d z \wedge d \bar{z}}{(1+z \bar{z})^{2}} .
$$

The double-cut of a generic $n$-point amplitude in the $K^{2}$-channel is defined as

$$
\Delta \equiv \int d^{4} \Phi A_{L}^{\mathrm{tree}}\left(\ell_{1}\right) A_{R}^{\mathrm{tree}}\left(\ell_{1}\right)
$$

where $A_{L, R}^{\text {tree }}$ are the tree-level amplitudes sitting at the two sides of the cut, see Fig.1. By using (2.5) for the LIPS, and (2.2) for the loop-momentum $\ell_{1}^{\mu}$, one has,

$$
\Delta=(1-2 \rho) \iint d z \wedge d \bar{z} \frac{A_{L}^{\text {tree }}(z, \bar{z}) A_{R}^{\text {tree }}(z, \bar{z})}{(1+z \bar{z})^{2}},
$$

\footnotetext{
${ }^{1}$ In terms of spinor variables that are associated to massless momenta, we can define $p^{\mu}=(1 / 2)\left\langle p\left|\gamma^{\mu}\right| p\right]$ and $q^{\mu}=(1 / 2)\left\langle q\left|\gamma^{\mu}\right| q\right]$, hence $\varepsilon_{+}^{\mu}=(1 / 2)\left\langle q\left|\gamma^{\mu}\right| p\right]$ and $\varepsilon_{-}^{\mu}=(1 / 2)\left\langle p\left|\gamma^{\mu}\right| q\right]$.
} 


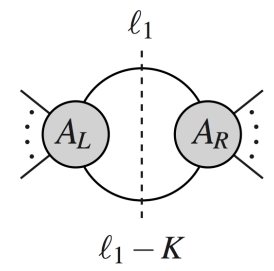

Figure 1: Double-cut of one-loop amplitude in the $K^{2}$-channel.

where the tree-amplitudes $A_{L}^{\text {tree }}$ and $A_{R}^{\text {tree }}$ are rational in $z$ and $\bar{z}$. Since $\rho$ is independent of $z$ and $\bar{z}$, its presence in the integrand is understood. By applying a special version of the so called Generalised Cauchy Formula also known as the Cauchy-Pompeiu Formula [28], one can write the two-fold integration in $z$ - and $\bar{z}$-variables appearing in Eq.(2.7) simply as a convolution of an unbounded $\bar{z}$-integral and a contour $z$-integral ${ }^{2}[26]$,

$$
\Delta=(1-2 \rho) \oint d z \int d \bar{z} \frac{A_{L}^{\text {tree }}(z, \bar{z}) A_{R}^{\text {tree }}(z, \bar{z})}{(1+z \bar{z})^{2}},
$$

where the product $A_{L}^{\text {tree }} A_{R}^{\text {tree }}$ is a rational function of $z$ and $\bar{z}$, and the integration contour has to be chosen as enclosing all the complex $z$-poles. The equivalence of Eq.(2.7) and Eq.(2.8) is due to Stokes' Theorem [26]. Accordingly, the double-cut $\Delta$ in (2.7) is the flux of a 2-form, corresponding to an integral over the complex tangent bundle of the Riemann sphere ${ }^{3}$, where the curvature 2-form is defined as $\Omega=1 /\left(1+|z|^{2}\right)^{2} d z \wedge d \bar{z}$.

\subsection{Coefficient of the 2-point function}

The formula in Eq.(2.8) can be intrgrated straightforwardly in two steps. To begin with the integration, we find a primitive with respect to $\bar{z}$, say $F$, by keeping $z$ as independent variable,

$$
F(z, \bar{z})=\int d \bar{z} \frac{A_{L}^{\text {tree }}(z, \bar{z}) A_{R}^{\text {tree }}(z, \bar{z})}{(1+z \bar{z})^{2}}
$$

so that $\Delta$ becomes,

$$
\Delta=(1-2 \rho) \oint d z F(z, \bar{z})
$$

Since $F$ is the primitive of a rational function, its general form can only contain two types of terms: a rational term and a logarithimc one,

$$
F(z, \bar{z})=F^{\mathrm{rat}}(z, \bar{z})+F^{\log }(z, \bar{z}) .
$$

The coefficient of a 2-point function in the $K^{2}$-channel will appear in $\Delta^{\text {rat }}$, namely the result of the Residue Theorem in $z$ applied only to $F^{\text {rat }}$. The choice of $p$ and $q$ specified in Eqs.(2.3) grants that there exists a pole at $z=0$ associated to the 2-point function in the $K^{2}$-channel, $I_{2}\left(K^{2}\right)$; while

\footnotetext{
${ }^{2}$ The roles of $z$ and $\bar{z}$ can be equivalently exchanged.

${ }^{3}$ In [26] it has been shown that the double-cut of the scalar 2-point function, $\Delta I_{2}=\int d^{4} \Phi$ amounts to the integral $\iint \Omega=-2 \pi i$. This result corresponds to the integration of the first Chern class, $(i / \pi) \iint \Omega=2$.
} 
the reduction of higher-point functions that have $I_{2}\left(K^{2}\right)$ as subdiagram can generate poles at finite $z$-values. The Residue Theorem has to be applied by reading all the residues in $z$, and substituting the corresponding complex-conjugate values where $\bar{z}$ appears. Since it can be shown [26] that the double-cut of the 2-point scalar function in the $K^{2}$-channel amounts to $\Delta I_{2}=-2 \pi i(1-2 \rho)$, the coefficient of the 2-point function can be finally extracted from the ratio,

$$
c_{2}=\frac{\Delta^{\mathrm{rat}}}{\Delta I_{2}}=-\left(\operatorname{Res}_{z=0} F^{\mathrm{rat}}(z, \bar{z})+\operatorname{Res}_{z \neq 0} F^{\mathrm{rat}}(z, \bar{z})\right),
$$

which will depend on $\rho$.

Recently, the method just described has been succesfully applied to the completion of the analytic calculation of the one-loop virtual corrections to $H+2$ jets via gluon fusion [29, 30]. ${ }^{4}$

\section{Optical Theorem and Berry's Phase}

In [27] the following observation was made. In the double-cut integral (2.7), we did not make any assumptions on the tree-level amplitudes sewn along the cut, thus providing a general framework to the integration method developed in [26]. If we now choose $A_{L}^{\text {tree }}=A_{m \rightarrow 2}^{* \text {,tree }}$, that is the conjugate scattering amplitude of a process $m \rightarrow 2$, and $A_{R}^{\text {tree }}=A_{n \rightarrow 2}^{\text {tree }}$, that is the amplitude of a process $n \rightarrow 2$, then $\Delta$ reads,

$$
\Delta=\int d^{4} \Phi A_{m \rightarrow 2}^{*, \text { tree }} A_{n \rightarrow 2}^{\text {tree }}=-i\left[A_{n \rightarrow m}^{\text {one-loop }}-A_{m \rightarrow n}^{* \text {,one-loop }}\right]=2 \operatorname{Im}\left\{A_{n \rightarrow m}^{\text {one-loop }}\right\}
$$

which is the definition of the two-particle discontinuity of the one-loop amplitude $A_{n \rightarrow m}^{\text {one-loop }}$ across the branch cut in the $K^{2}$-channel, corresponding to the field-theoretic version of the Optical Theorem for one-loop Feynman amplitudes. On the other side, because of Stokes' Theorem in (2.7, 2.8), one has,

$$
\Delta=(1-2 \rho) \iint d z \wedge d \bar{z} \frac{A_{m \rightarrow 2}^{*, \text { tree }} A_{n \rightarrow 2}^{\text {tree }}}{(1+z \bar{z})^{2}}=(1-2 \rho) \oint d z \int d \bar{z} \frac{A_{m \rightarrow 2}^{* \text {,tree }} A_{n \rightarrow 2}^{\text {tree }}}{(1+z \bar{z})^{2}},
$$

which provides a geometrical interpretation of the imaginary part of one-loop scattering amplitudes, as a flux of a complex 2-form through a surface bounded by the contour of the $z$-integral (the contour should enclose all the poles in $z$ exposed in the integrand after the integration in $\bar{z}$ [26]).

Given the equivalence of (3.1) and (3.2), a correspondence between the imaginary part of scattering amplitudes and the anholonomy of Berry's phase does emerge, since the latter is indeed defined as the flux of a 2-form in presence of curved space [1,2]. In this context, one could establish a parallel description between the Aharonov-Böhm (AB) effect [31] and the double-cut of one-loop Feynman integrals. Accordingly, let us follow the evolution in Fig.1 from the left to the right. The two particles produced in the $A_{L}$-scattering, going around the loop and initiating the $A_{R}$-process, at the $A_{R}$-interaction point would experience a phase-shift due to the non-trivial geometry in effective momentum space induced by the on-shell conditions. As in the AB-effect, the anholonomy phaseshift is a consequence of Stokes' Theorem, and here it corresponds to the imaginary part of the one-loop Feynman amplitude.

\footnotetext{
${ }^{4}$ See also S. Badger, and C. Williams in these proceedings.
} 


\section{References}

[1] M. V. Berry, Proc. Roy. Soc. Lond. A 392 (1984) 45.

[2] A. D. Shapere and F. Wilczek (ed.), Adv. Ser. Math. Phys. 5 (1989) 1.

[3] L. D. Landau, Nucl. Phys. 13, 181 (1959). R. E. Cutkosky, J. Math. Phys. 1, 429 (1960). R. J. Eden, P. V. Landshoff, D. I. Olive, J. C. Polkinghorne, The Analytic S Matrix, Cambridge University Press, 1966. M. J. G. Veltman, Physica 29 (1963) 186. E. Remiddi, Helv. Phys. Acta 54 (1982) 364.

[4] R. C. Hwa, and V. L. Teplitz, Mathematical Physics Monographs, W. A. Benjamin Inc., 1966.

[5] Z. Bern, L. J. Dixon, D. C. Dunbar and D. A. Kosower, Nucl. Phys. B 425 (1994) 217; Nucl. Phys. B 435 (1995) 59.

[6] G. Passarino and M. J. G. Veltman, Nucl. Phys. B 160 (1979) 151.

[7] G. Ossola, C. G. Papadopoulos and R. Pittau, Nucl. Phys. B 763, 147 (2007); JHEP 0707, 085 (2007).

[8] R. K. Ellis, W. T. Giele and Z. Kunszt, JHEP 0803 (2008) 003.

[9] W. T. Giele, Z. Kunszt and K. Melnikov, JHEP 0804 (2008) 049.

[10] C. F. Berger et al., Phys. Rev. D 78 (2008) 036003.

[11] R. Britto, F. Cachazo and B. Feng, Nucl. Phys. B 725, 275 (2005).

[12] P. Mastrolia, Phys. Lett. B 644, 272 (2007).

[13] T. Binoth, G. Heinrich, T. Gehrmann and P. Mastrolia, Phys. Lett. B 649, 422 (2007).

[14] D. Forde, Phys. Rev. D 75, 125019 (2007).

[15] N. E. J. Bjerrum-Bohr, D. C. Dunbar and W. B. Perkins, JHEP 0804, 038 (2008).

[16] W. B. Kilgore, arXiv:0711.5015 [hep-ph].

[17] S. D. Badger, JHEP 0901 (2009) 049.

[18] R. Britto, E. Buchbinder, F. Cachazo and B. Feng, Phys. Rev. D 72, 065012 (2005).

[19] R. Britto, B. Feng and P. Mastrolia, Phys. Rev. D 73, 105004 (2006).

[20] C. Anastasiou, R. Britto, B. Feng, Z. Kunszt and P. Mastrolia, Phys. Lett. B 645, 213 (2007); JHEP 0703, 111 (2007).

[21] R. Britto and B. Feng, Phys. Rev. D 75, 105006 (2007); JHEP 0802, 095 (2008).

[22] R. Britto, B. Feng and P. Mastrolia, Phys. Rev. D 78 (2008) 025031.

[23] R. Britto, B. Feng and G. Yang, JHEP 0809 (2008) 089.

[24] E.W.N. Glover and C. Williams, JHEP 0812 (2008) 067.

[25] R. Britto and B. Feng, Phys. Lett. B 681, 376 (2009).

[26] P. Mastrolia, Phys. Lett. B 678 (2009) 246.

[27] P. Mastrolia, to appear in Lett. Math. Phys., arXiv:0906.3789.

[28] M. J. Ablowitz, and A. S. Fokas, Complex Variables, Cambridge Texts in Applied Mathematics, 2003, 2nd Edition.

[29] S. Badger, E. W. Nigel Glover, P. Mastrolia and C. Williams, to appear in JHEP, arXiv:0909.4475.

[30] S. Badger, J. M. Campbell, R. K. Ellis and C. Williams, JHEP 0912 (2009) 035.

[31] Y. Aharonov and D. Bohm, Phys. Rev. 115 (1959) 485. 